\title{
Use of B-complex vitamins and olfactory training for treating COVID-19-related anosmia
}

\author{
Yethindra Vityala ${ }^{1}$, Shirin Zhumabaeva ${ }^{1}$, Baktygul Imankulova ${ }^{1}$, Alina Kurmanalieva ${ }^{1}$, and \\ Sagynali Mamatov ${ }^{2}$ \\ ${ }^{1}$ International Higher School of Medicine, International University of Kyrgyzstan \\ ${ }^{2}$ I K Akhunbaev Kyrgyz State Medical Academy
}

April 1, 2021

\begin{abstract}
A 42-year-old male patient was diagnosed with coronavirus disease 2019. His symptoms improved 2 weeks after lopinavir therapy (400 mg every 12 hours). However, he was subsequently diagnosed with complete anosmia. Magnetic resonance brain imaging showed no abnormalities. We prescribed B-complex vitamins and olfactory training. Forty days later, he recovered.
\end{abstract}

\section{Use of B-complex vitamins and olfactory training for treating COVID-19-related anosmia}

Yethindra Vityala $^{1}$, Shirin Zhumabaeva ${ }^{2}$, Baktygul Imankulova ${ }^{3}$, Alina Kurmanalieva ${ }^{4}$, Sagynali Mamatov ${ }^{5}$

${ }^{1}$ Department of Pathology, International Higher School of Medicine, International University of Kyrgyzstan, Bishkek, Kyrgyzstan

${ }^{2}$ Department of Special Clinical Disciplines, International Higher School of Medicine, International University of Kyrgyzstan, Bishkek, Kyrgyzstan

${ }^{3}$ Department of Public Health, International Higher School of Medicine, International University of Kyrgyzstan, Bishkek, Kyrgyzstan

${ }^{4}$ Department of Infectious Diseases, International Higher School of Medicine, International University of Kyrgyzstan, Bishkek, Kyrgyzstan

${ }^{5}$ Department of Hospital Internal Medicine, Occupational Pathology with a Course of Hematology, I.K. Akhunbaev Kyrgyz State Medical Academy, Bishkek, Kyrgyzstan

Running title: Treatment for COVID-19-related anosmia

*Corresponding author: Vityala Yethindra, Department of Pathology, International Higher School of Medicine, International University of Kyrgyzstan, Bishkek, Kyrgyzstan, Email:yethindravityala10@gmail.com, Contact: +919121925658

\section{ABSTRACT}

A 42-year-old male patient was diagnosed with coronavirus disease 2019. His symptoms improved 2 weeks after lopinavir therapy (400 mg every 12 hours). However, he was subsequently diagnosed with complete anosmia. Magnetic resonance brain imaging showed no abnormalities. We prescribed B-complex vitamins and olfactory training. Forty days later, he recovered.

Keywords: SARS-CoV-2, COVID-19, Anosmia, Lopinavir 


\section{Key clinical message}

At present, as there is no proper treatment for COVID-19-related anosmia, we recommend the use of Bcomplex vitamins and olfactory training for treating COVID-19-related anosmia.

\section{CASE SUMMARY}

Anosmia tends to manifest during the early stages of coronavirus disease 2019 (COVID-19). ${ }^{1}$ A 42-year-old male patient was hospitalized for intermittent fever, dry cough, and malaise. On the third day of hospitalization, real-time reverse transcriptase-polymerase chain reaction analysis of his nasopharyngeal samples yielded a positive result for severe acute respiratory syndrome coronavirus 2 ; thus, the patient was diagnosed with COVID-19 and treated with $400 \mathrm{mg}$ lopinavir every $12 \mathrm{~h}$ for 2 weeks. After 1 week of hospitalization, the patient reported losing his sense of smell in both nasal cavities. Based on olfactory function tests, he was diagnosed with complete anosmia and olfactory function tests evaluate the degree of anosmia more objectively, ${ }^{2}$ which is helpful during the follow-up of patients to evaluate treatment response. Magnetic resonance brain imaging showed no abnormalities (Figure 1). Thereafter, treatment with $5000 \mathrm{IU}$ of Citoneurin (Vitamin B1 [thiamine hydrochloride], $100 \mathrm{mg}$ /day; Vitamin B6 [pyridoxine hydrochloride], $100 \mathrm{mg} /$ day; Vitamin B12 [cyanocobalamin], $5000 \mathrm{mcg} /$ day) orally and olfactory training, which consists of inhaling identified flasks containing cinnamon, cloves, and lavender for 15 minutes/day were prescribed. Repeated exposure to an odorant may modulate the regenerative capacity of the olfactory receptors. ${ }^{3}$ Subjective signs of anosmia began to improve 12 days later. Two weeks after lopinavir therapy, the patient recovered from most symptoms of COVID-19; and forty days after initiation of B-complex vitamins and olfactory training, he recovered from anosmia. We conclude that B-complex vitamins and olfactory training may be effective for the treatment of COVID-19-related anosmia.

\section{ACKNOWLEDGMENTS}

The authors acknowledge their patient for kind cooperation and for providing consent.

\section{FINANCIAL SUPPORT}

No financial support was received for the study.

\section{CONFLICT OF INTEREST}

The authors declare no conflicts of interest.

\section{AUTHOR CONTRIBUTIONS}

Conception, design of the work, manuscript preparation, and data acquisition: Yethindra Vityala, Shirin Zhumabaeva, Baktygul Imankulova, Alina Kurmanalieva, Sagynali Mamatov, Clinical management: Yethindra Vityala, Shirin Zhumabaeva, Sagynali Mamatov, and Manuscript preparation and data acquisition: Yethindra Vityala, Alina Kurmanalieva, Baktygul Imankulova.

\section{DATA AVAILABILITY STATEMENT}

Data are available from the corresponding author upon reasonable request.

\section{REFERENCES}

1. Giacomelli A, Pezzati L, Conti F, et al. Self-reported olfactory and taste disorders in patients with severe acute respiratory coronavirus 2 infection: a cross-sectional study. Clin Infect Dis. 2020; 71: 889-90.

2. Hummel T, Sekinger B, Wolf SR, et al. 'Sniffin' sticks: olfactory performance assessed by the combined testing of odor identification, odor discrimination and olfactory threshold. Chem Senses. 1997; 22: 39-52.

3. Damm M, Pikart LK, Reimann H, et al. Olfactory training is helpful in postinfectious olfactory loss: a randomized, controlled, multicenter study. Laryngoscope. 2014; 124: 826-31. 


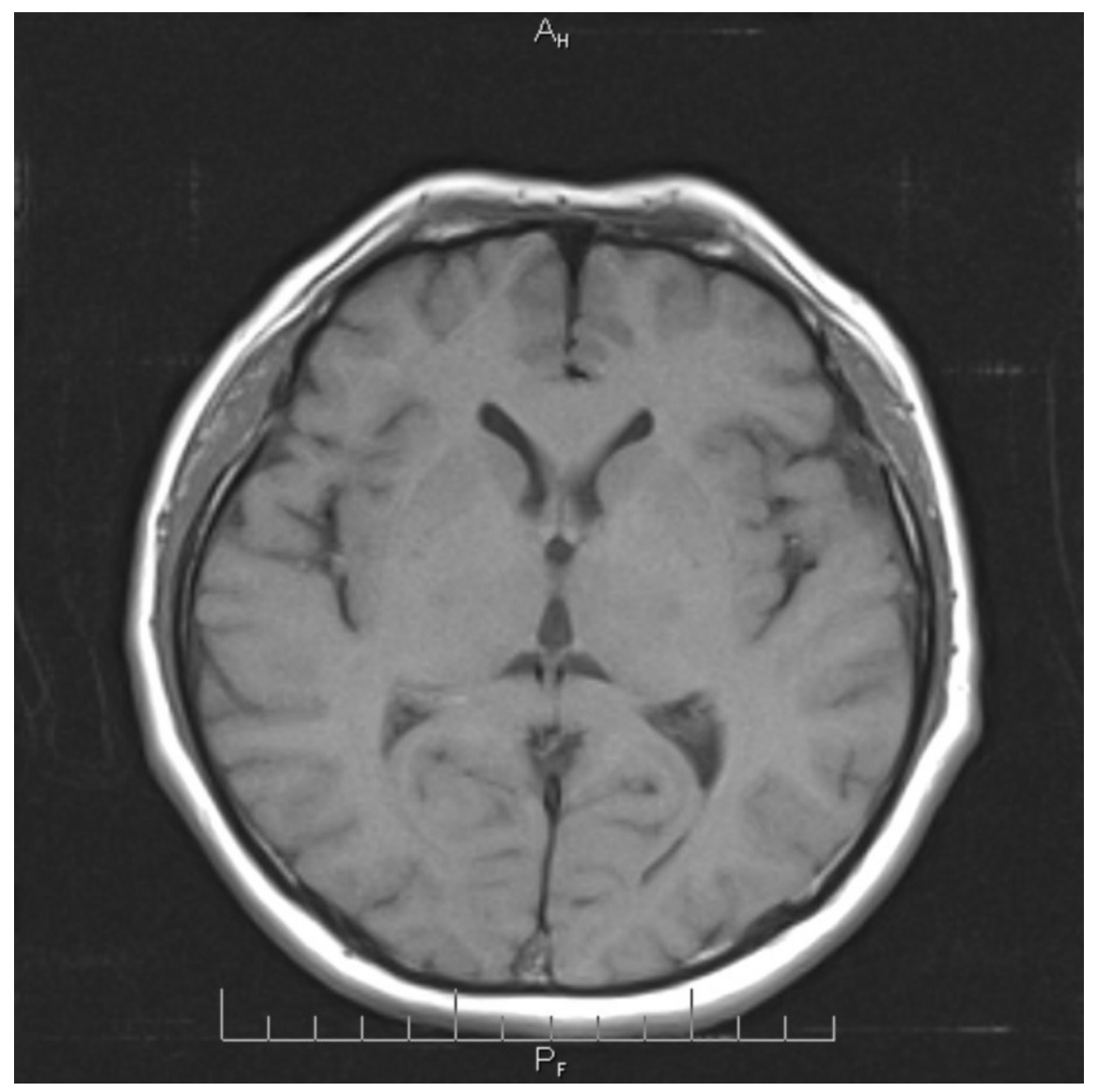

